Copyright (C) 2014 IEEE. Personal use of this material is permitted. Permission from IEEE must be obtained for all other uses, in any current or future media, including reprinting/republishing this material for advertising or promotional purposes, creating new collective works, for resale or redistribution to servers or lists, or reuse of any copyrighted component of this work in other works. 


\section{A Novel Queue Length Aware Distributed Link Scheduler for Multi-Transmit Receive Wireless Mesh Networks}

\author{
Yuanhuizi Xu, Kwan-Wu Chin, Raad Raad \\ School of Electrical, Computer and Telecommunications Engineering \\ University of Wollongong, NSW, Australia \\ Email: yx879@uowmail.edu.au, \{kwanwu,raad\}@uow.edu.au
}

\author{
Sieteng Soh \\ Department of Computing \\ Curtin University of Technology, WA, Australia \\ Email: s.soh@curtin.edu.au
}

\begin{abstract}
Next generation Wireless Mesh Networks (WMNs) will require a link scheduler that exploits the full advantage of Multi-Transmit-Receive (MTR) commuication. To this end, we design a distributed link scheduler called Voting-ALGO that is aware of queue lengths and uses the celebrated max weight policy to achieve $100 \%$ throughput.
\end{abstract}

\section{INTRODUCTION}

In Multi-Transmit-Receive Wireless Mesh Networks (MTR WMNs), whereby nodes are equipped with adaptive arrays, nodes can transmit simultaneously to (SynTx), or receive simultaneously from (SynRx) their respective neighbouring nodes. Hence, a key constraint of any channel access or link scheduler over such WMNs must adhere to this so called MixTx-Rx capability.

To this end, we require a sheduler that addresses the following problem: given an MTR WMN with stochastic arrival rates, design a throughput optimal, distributed algorithm that activates the highest weighted links. Since the seminal contribution of Tassiulas et al. [1], who proposed a centralized maximum weight policy that is shown to be throughput optimal using only topological and queue length information, a number of researchers have sought distributed schedulers; see [2]. These schedulers, however, assume a $k$ hop interference model, and thus do not consider the MTR capability of nodes.

To date, only a handful of works have investigated centralized and distributed link scheduling algorithms for MTR WMNs. The authors of [3] proposed a distributed solution that allows nodes to hold the channel longer based on traffic demand. A key observation is that this algorithm has not considered queue lengths. Indeed, only Bao et al. [4], who proposed receive-oriented multiple access (ROMA) scheme, have considered link weights, whereby a heavier weight corresponds to a higher probability of activation. However, ROMA only provides four different weight values and no solution is presented on how they can be adjusted to achieve queue stability. In contrast, our proposed algorithm, called VotingALGO, provides a deterministic method that schedules the highest weighted links in each transmission slot. Moreover, it provides the following advantages: 1) it does not have excessive communication overheads associated with data collection,
2) it uses queue lengths to evenly split nodes into transmitter and receivers at each time slot. Experimental results show Voting-ALGO achieves higher capacity than prior distributed link schedulers designed for MTR WMNs.

Next, we outline our network model, and formalize the problem in Section III. Voting-ALGO, is outlined in Section IV. Section V presents our evaluation and results, followed by our conclusions in Section VI.

\section{NeTWORK MODEL}

Consider a WMN represented by a directed graph $G(V, E)$, where $V=\{1, \ldots, n\}$ is the set of nodes and $E=\{(u, v) \in$ $E \mid u, v \in V\}$ is the set of directed links. Each node has a unique ID. All nodes are equipped with $b \geq 1$ directional antennas that share a single channel. We assume $b_{u} \geq|N(u)|$ for all nodes, where $N(u)$ is the set of neighbors for node $u$. Each node has a transmission range of $r$. We consider a link exists if node $u$ and $v$ are within each other's transmission range. Thus, two nodes that are located within a distance smaller than the transmission range are regarded as neighbors. At any point in time, a node $u$ can transmit to or receive from $|N(u)|$ neighbors at the same time, but it cannot receive and transmit simultaneously because of side-lobes. We will refer to this as the no Mix-Tx-Rx interference constraint. In addition, we consider the 1-hop traffic model where all routes consist of one link. In this respect, we will denote the transmitting end of a link as source, and the corresponding end as receiver.

We assume time is divided into slots of equal length, denoted by $t$. The packets are of unit length. In this paper, we consider the Bernoulli process with parameter $\lambda$, which is known as the arrival rate. We use a $0-1$ vector with dimension $|E|$, and denoted as $A(t)$, to represent a packet arrival at each queue/link at the beginning of slot $t$. We assume the arrivals are independent identically distributed. The arriving packet is stored in the queue associated to said link. The vector $A(t)$ is calculated based on the arrival rate $\lambda$. Let $Q_{u, v}(t) \geq 0$ represent the number of packets in the queue of link $(u, v)$ at time $t$. Thus, the queue size vector is denoted by $Q(t)=\left[Q_{u, v}(t)\right]$. The vector $D(t) \subseteq\{0,1\}^{|E|}$ denotes transmitting links at the end of slot $t$ that adhere to the no Tx-Rx constraint. Thus a link $(u, v)$ is active if $D_{u v}(t)=1$. 
TABLE I

Messages USED IN Voting-ALGO

\begin{tabular}{|l|l|}
\hline Message & Description \\
\hline$Q_{s, d}$ & The queue-length of link $(s, d)$. \\
\hline$I N q(v)$ and $O U T q(v)$ & $\begin{array}{l}\text { The total number of incoming and outgoing } \\
\text { packets at node } v \text { respectively. }\end{array}$ \\
\hline INsetT and INsetR & $\begin{array}{l}\text { Notification messages sent by nodes in setT } \\
\text { and setR respectively to inform their neigh- } \\
\text { bors which set they joined. }\end{array}$ \\
\hline $\operatorname{Vote}\left\{s \rightarrow d, Q_{s, d}\right\}$ & $\begin{array}{l}\text { Vote message sent by nodes in setT or setR } \\
\text { to their neighbors in set } U .\end{array}$ \\
\hline$I N q^{\prime}(v)$ and $O U T q^{\prime}(v)$ & $\begin{array}{l}\text { The number of incoming and outgoing pack- } \\
\text { ets from neighbors in setT or to neighbors } \\
\text { in setR at node } v \text { based on received vote } \\
\text { messages. }\end{array}$ \\
\hline
\end{tabular}

Consequently, the dynamic queue length of the network model can be described as $Q(t)=A(t)+Q(t-1)-D(t-1)$.

\section{Problem Definition}

We now formalize the problem at hand. Given queue length information, design a throughput optimal distributed algorithm. Formally, $\arg \max _{D}(T) \lambda$ s.t. $\lambda \in \Lambda$ (or $\arg \max _{D}(T) \sum Q(T) * D(T)$ s.t. $\lambda \in \Lambda$, where $T$ denotes the throughput). As mentioned earlier, the Max Weight policy is throughput optimal. However, it is centralized and requires instantaneous queue length information, which is not practical in WMNs. In particular, the centralized policy will incur many rounds of requests, and signalling overheads as well as propagation delays, which result in stale queue size information. These limitations thus motivate the design of a distributed algorithm that is throughput optimal.

\section{The Distributed Policy}

We now expound a distributed policy called Voting-ALGO. We assume each node has a distinct ID. The basic idea is as follows. We divide each time slot into two sub-slots: control and data. The first sub-slot is used for scheduling, during which time each node determines whether it is in transmitting set set $T$ or receiving set setR. The second sub-slot data is used for packets transmission. That is, at such time, all the nodes in set $T$ transmit one packet to each of their neighbors that are in setR. Each control sub-slot is further divided into a number of mini-slots. In the mini-slots, nodes exchange control messages listed in Table I. Note, we denote the vote message as "Vote $\left\{s \rightarrow d, Q_{s, d}\right\}$ ", where $s$ and $d$ represent the source and destination nodes of link $(s, d)$, and $Q_{s, d}$ is the queue length of link $(s, d)$.

Our Voting-ALGO consists of three key stages: initialize, notify and vote. In the first stage, as shown in Algorithm 1, the goal is to select the first batch of nodes that are to join setT or setR. All other nodes move into set $U$, where $\operatorname{set} U=V-$ set $T$ - set $R$. Algorithm 2 outlines the pseudocode performing notify, whereby nodes that have entered $\operatorname{set} T$ or setR in Stage1 inform their neighbors which set they have joined by sending the message "INsetT" and "INsetR". After that, nodes proceed to the key stage vote which is demonstrated in Algorithm 3. The main idea is to determine which set, i.e., set $T$ or $\operatorname{set} R$,
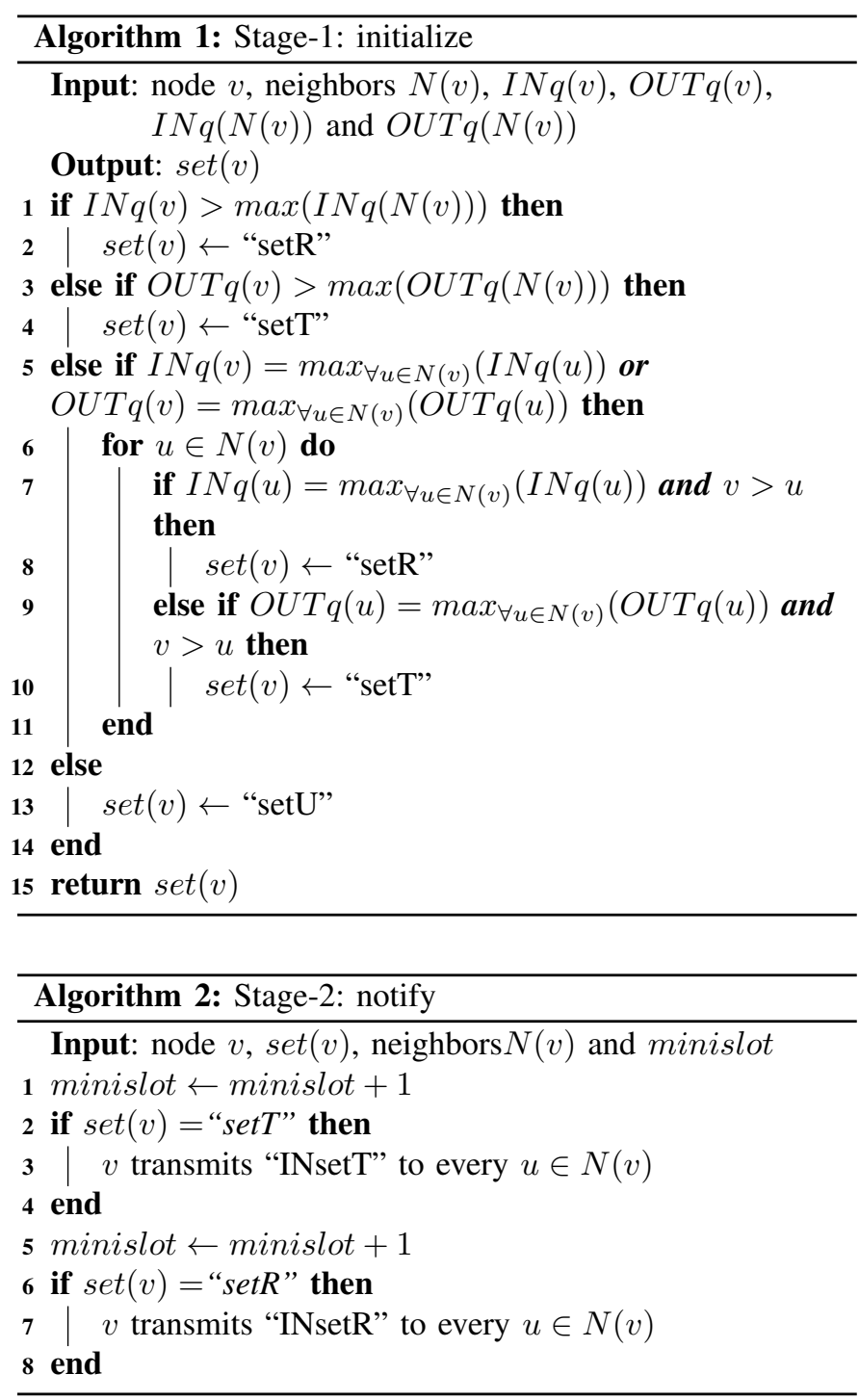

should nodes in set $U$ join based on the votes cast by their neighbors. To achieve this, after receiving all the votes, each node calculates its $I N q^{\prime}$ and $O U T q^{\prime}$ which represents the total number of votes that elect the node a $\operatorname{set} R$ or a set $T$ node. If $I N q^{\prime}$ is greater than or equal to $O U T q^{\prime}$, the node is included in setR. Otherwise, it joins setT. From this point onwards, Stage- 2 and Stage- 3 is carried out alternately until there is no node left in set $U$.

\section{Evaluation}

In order to investigate the performance of Voting-ALGO, we use MatGraph [5], a Matlab toolkit that works with simple graphs. Our Matlab experiments are conducted over topologies with 50 nodes, whereby the degree for each node is fixed and equals to five. We compare Voting-ALGO against link based centralised algorithm (LBC-ALGO), node based centralised algorithm (NBC-ALGO), JazzyMAC [3] and ROMA [4]. Both LBC-ALGO and NBC-ALGO require the global queue length information. LBC-ALGO selects the set of heaviest-weighted, 


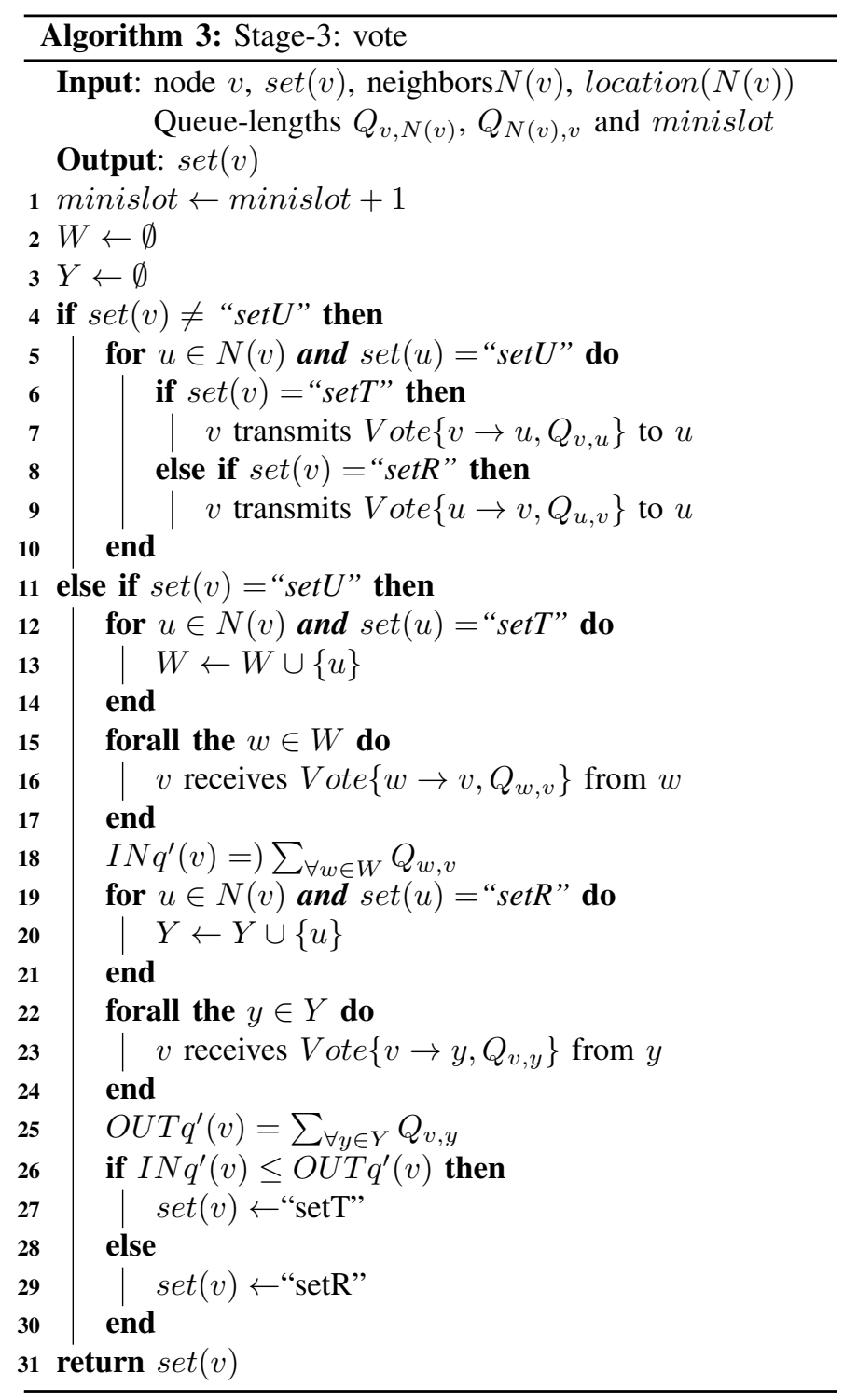

non-interfering links at every time slot to transmit, where weight is the length of queue of each link. While the queue length variable used in NBC-ALGO denotes the sum of the queue length of every outgoing links at each node. In every slot, the nodes with longest queue length are placed into set $T$ and their neighbours into setR.

In the experiments, we assume all traffic traverse a single hop. A packet arrival occurs with probability $\lambda$ at the beginning of a time slot. The arrivals are independent and identically distributed according to a Bernoulli process.

Figure 1 shows the mean total queue backlog summed over all links as the arrival rate $\lambda$ increases. The error bars shown indicate $95 \%$ confidence interval of the mean value, which represents the true queue length. When $\lambda$ approaches a certain limit, the average total backlog will increase to infinity. This limit is viewed as the boundary of the capacity region. The results are collected after 100,000 time slots.

From Figure 1, we can see that, generally, Voting-ALGO is superior to other algorithms as it ensures all queues are stable with the same traffic load. We see that Voting-ALGO achieves the same capacity region as LBC-ALGO. This result indicates that the performance of Voting-ALGO is approximately similar to centralized approaches. In other words, Voting-ALGO is throughput optimal.

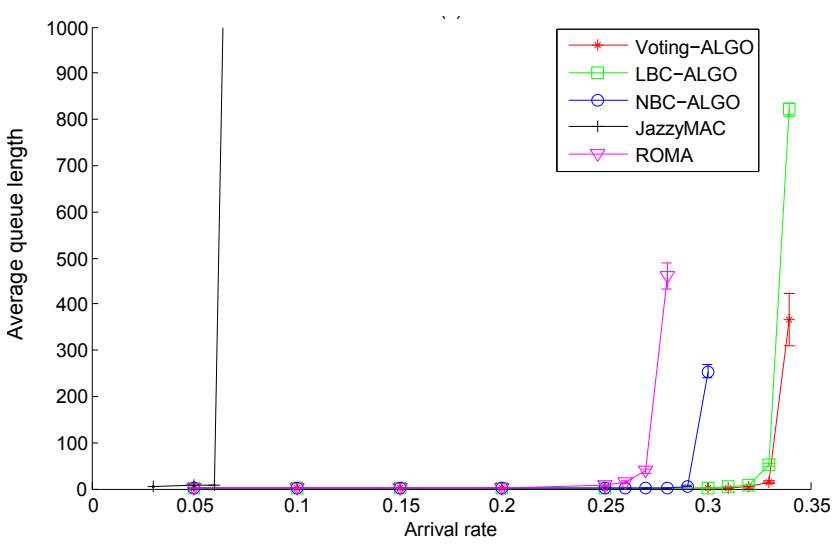

Fig. 1. Queue length under increasing arrival rate with $95 \%$ confidence interval

\section{CONCLUSION}

This paper has presented a novel queue aware distributed link scheduler for MTR WMNs whereby nodes have the capability to form multiple links simultaneously. Experiment results show the throughput achieved by Voting-ALGO is two to nine times higher than that of JazzyMAC. An immediate future work is to further reduce signaling messages and to consider the case when the number of directional antennas is less than the number of neighbors.

\section{REFERENCES}

[1] L. Tassiulas and A. Ephremides, "Stability properties of constrained queueing systems and scheduling policies for maximum throughput in multihop radio networks," IEEE Transactions on Automatic Control, vol. 37, no. 12, pp. 1936-1948, 1992.

[2] X. Lin and N. Shroff, "The impact of imperfect scheduling on cross layer control in wireless networks," IEEE Transactions on Networking, vol. 14, pp. 302-315, Apr. 2006.

[3] S. Nedevschi, R. Patra, S. Surana, S. Ratnasamy, L. Subramanian, and E. Brewer, "An adaptive, high performance mac for long-distance multihop wireless networks," in ACM MOBICOM, (New York, NY, USA), pp. $259-270,2008$.

[4] B. Lichun and J. Garcia-Luna-Aceves, "Transmission scheduling in ad hoc networks with directional antennas," in ACM MOBICOM, (New York, NY, USA), 2002.

[5] E. R. Scheinerman, "Matgraph: a MATLAB toolbox for graph theory," Department of applied mathematics and statistics, the Johns Hopkins University, Baltimore, Maryland, pp. 1- 7, 2008. 